\title{
Interplay of Coulomb blockade and Aharonov-Bohm resonances in a Luttinger liquid
}

\author{
Jari M. Kinaret ${ }^{1}$, Mats Jonson ${ }^{1}$, Robert I. Shekhter ${ }^{1}$, Sebastian Eggert ${ }^{2}$ \\ ${ }^{1}$ Department of Applied Physics and ${ }^{2}$ Institute of Theoretical Physics, \\ Chalmers University of Technology and Göteborg University, S-41296 Göteborg, Sweden
}

\begin{abstract}
We consider a ring of strongly interacting electrons connected to two external leads by tunnel junctions. By studying the positions of conductance resonances as a function of gate voltage and magnetic flux the interaction parameter $g$ can be determined experimentally. For a finite ring the minimum conductance is strongly influenced by device geometry and electron-electron interactions. In particular, if the tunnel junctions are close to one another the interaction-related orthogonality catastrophe is suppressed and the valley current is unexpectedly large.
\end{abstract}


When the size of an electronic system is reduced, a rich variety of new "mesoscopic" phenomena becomes experimentally observable. Some of the new phenomena are essentially classical, owing their existence to the granularity of the electric charge and the system size -dependence of various energy scales. The most widely studied example of this type is Coulomb blockade. A different category of mesoscopic phenomena is entirely quantum mechanical, and is due to the fact that the phase coherence length at low temperatures is comparable to the system size, giving rise to a number of interference effects. A particular example is the existence of a persistent current in the ground state of a mesoscopic ring. In this Letter we study the interplay between two mesoscopic phenomena, Coulomb blockade and Aharonov-Bohm interference, using an exactly solvable model.

We consider a system consisting of a small ring of interacting electrons connected to two non-interacting leads by tunnel junctions. The tunnel junctions are at positions $x_{L}$ and $x_{R}$, respectively. The ring is capacitively coupled to an external gate electrode and may be pierced by a magnetic flux. We consider a small AC voltage applied to the right lead and wish to evaluate the current at the left junction. A straightforward application of Kubo formula [1] yields the current

$$
\left\langle I_{L}(t)\right\rangle=-i \frac{V(t)}{\hbar \Omega} \int_{-\infty}^{t} d t^{\prime} e^{-i \Omega\left(t^{\prime}-t\right)} \operatorname{Tr}\left\{\hat{\rho}_{G}\left[\hat{I}_{R}\left(t^{\prime}\right), \hat{I}_{L}(t)\right]\right\}
$$

where $V(t)=V_{0} e^{-i \Omega t}$ is the applied voltage and $\hat{\rho}_{G}$ is the equilibrium density matrix. The quantity on the right hand side is recognized as the retarded current-current correlation function. It is most readily evaluated in imaginary time.

The Hamiltonians for the leads and the connection between the leads and the ring are given by

$$
\begin{aligned}
H_{L} & =\sum_{k} \epsilon_{k L} c_{L}^{\dagger}(k) c_{L}(k) \\
H_{L T} & =t_{L} c_{L}^{\dagger}\left(x_{L}\right) \psi\left(x_{L}\right)+t_{L}^{*} \psi^{\dagger}\left(x_{L}\right) c_{L}\left(x_{L}\right)
\end{aligned}
$$

where $c_{L}$ are operators on the left lead and $\psi$ are operators on the ring. The Hamiltonians for the right lead are defined analogously. Thus, we take the leads to be non-interacting and couple them to the ring with tunnel junctions at positions $x_{L}$ and $x_{R}$. To calculate the current-current correlation function we define the generating functional $Z\left[J_{L}, J_{R}\right]=$ $\operatorname{Tr} \exp \left[-\beta H-\frac{1}{\hbar} \int_{0}^{\hbar \beta} d \tau\left(J_{L}(\tau) I_{L}(\tau)+J_{R}(\tau) I_{R}(\tau)\right)\right]$ and integrate out the free fermions in the leads. That yields

$$
\begin{aligned}
& Z\left[J_{L}, J_{R}\right]=Z_{L} Z_{R} \operatorname{Tr} \exp \left\{-\beta H_{\text {ring }}-\frac{1}{\hbar^{2}} \int_{0}^{\hbar \beta} d \tau \int_{0}^{\hbar \beta} d \tau^{\prime}\right. \\
& {\left[\left|t_{L}\right|^{2}\left(1-\frac{i e}{\hbar} J_{L}(\tau)\right)\left(1+\frac{i e}{\hbar} J_{L}\left(\tau^{\prime}\right)\right) \psi^{\dagger}\left(\tau, x_{L}\right) G_{L}\left(\tau-\tau^{\prime} ; x_{L}, x_{L}\right) \psi\left(\tau^{\prime}, x_{L}\right)\right.} \\
& \left.\left.+\left|t_{R}\right|^{2}\left(1+\frac{i e}{\hbar} J_{R}(\tau)\right)\left(1-\frac{i e}{\hbar} J_{R}\left(\tau^{\prime}\right)\right) \psi^{\dagger}\left(\tau, x_{R}\right) G_{R}\left(\tau-\tau^{\prime} ; x_{R}, x_{R}\right) \psi\left(\tau^{\prime}, x_{R}\right)\right]\right\}
\end{aligned}
$$

where $Z_{L / R}$ and $G_{L / R}\left(\tau ; x, x^{\prime}\right)$ are the partition functions and free fermion propagators in the leads, and $H_{\text {ring }}$ is the Hamiltonian for an isolated ring. The imaginary time ordered correlation function $\chi\left(\tau_{1}-\tau_{2}\right)=-\left\langle T_{\tau}\left(I_{L}\left(\tau_{1}\right) I_{R}\left(\tau_{2}\right)\right)\right\rangle$ is obtained by differentiating $Z\left[J_{L}, J_{R}\right]$ with respect to $J_{L}$ and $J_{R}$. To simplify the notation we introduce the four-operator expectation 
value $A\left(\tau_{1}, \tau, \tau_{2}, \tau^{\prime}\right)=A\left(\tau_{1}-\tau_{2}, \tau_{1}-\tau, \tau_{2}-\tau^{\prime}\right)=\left\langle T_{\tau}\left(\psi^{\dagger}\left(\tau, x_{L}\right) \psi\left(\tau_{1}, x_{L}\right) \psi^{\dagger}\left(\tau_{2}, x_{R}\right) \psi\left(\tau^{\prime}, x_{R}\right)\right)\right\rangle$ and its Fourier transform $A\left(i \omega_{n}, i \omega_{n}^{\prime}, i \omega_{n}^{\prime \prime}\right)$. To the lowest nonzero order in the tunneling matrix elements $\chi$ is given by

$$
\begin{aligned}
\chi\left(i \Omega_{n}\right) & =\frac{e^{2}}{\hbar^{4}}\left|t_{L} t_{R}\right|^{2} \frac{1}{(\hbar \beta)^{2}} \sum_{i \omega_{n}, i \omega_{n}^{\prime}}\left\{A\left(i \Omega_{n},-i \omega_{n},-i \omega_{n}^{\prime}\right)\right. \\
& \left.\times\left[G_{L}\left(i \omega_{n}-i \Omega_{n} ; x_{L}, x_{L}\right)-G_{L}\left(i \omega_{n} ; x_{L}, x_{L}\right)\right]\left[G_{R}\left(i \omega_{n}^{\prime} ; x_{R}, x_{R}\right)-G_{R}\left(i \omega_{n}^{\prime}+i \Omega_{n} ; x_{R}, x_{R}\right)\right]\right\}
\end{aligned}
$$

If we regard the leads as infinite (rather than semi-infinite), the propagators in the leads are easy to evaluate and yield $G_{L}\left(i \omega_{n} ; x_{L}, x_{L}\right)=-i \frac{\hbar}{2} D_{L}\left(\epsilon_{F}\right) \operatorname{sign}\left(\omega_{n}\right)$ where $D_{L}(\epsilon)$ is the density of states in the left lead.

To evaluate the four-operator product $A\left(i \omega_{n}, i \omega_{n}^{\prime}, i \omega_{n}^{\prime \prime}\right)$ we must specify the Hamiltonian for the ring. We choose to work with the simplest exactly solvable interacting model, the spinless Luttinger model. In the bosonized form the Hamiltonian reads [2]

$$
H_{\text {ring }}=\frac{\pi \hbar}{2 L}\left[\frac{v}{g}\left(\hat{N}-N_{0}\right)^{2}+g v\left(\hat{J}-J_{0}\right)^{2}\right]+\sum_{q \neq 0} \hbar v|q| b_{q}^{\dagger} b_{q}
$$

where $\hat{N}$ and $\hat{J}$ are zero modes associated with the total charge and total current. Since the numbers of clockwise and counterclockwise moving electrons on the ring must both be integers, the quantum numbers $N$ and $J$ must satisfy $(-1)^{N}=(-1)^{J}$. The gate voltage and magnetic flux determine the parameters $N_{0}=C V_{g} / e$ and $J_{0}=2 \Phi / \Phi_{0}$ which in turn determine the ground state charge and current. The parameter $g$ is a measure of the interaction strength, and equals one for non-interacting electrons [3]. For future use we also define the shorthand notation $\gamma=\frac{1}{2}\left(g+g^{-1}\right)-1$ which vanishes in the non-interacting limit.

Due to time-ordering the exact expression for $A$ is quite complicated although in principle straightforward. Since we consider only the lowest order in the tunneling Hamiltonian, our analysis is valid only sufficiently far from the conductance resonances. Therefore, we can assume that the ground state is separated from the excited states by an energy gap $\delta \epsilon$ that is larger than $k_{B} T$. This approximation is basically similar to the one used by Fazio and co-workers [4 for an interacting ring connected to superconducting leads. We also neglect events with all four imaginary times approximately equal since their contribution is negligible. That allows us to evaluate $A$ in an approximate fashion, and gives

$$
\begin{aligned}
& A\left(i \Omega_{n},-i \omega_{n},-i \omega_{n}^{\prime}\right) \approx \hbar \beta\left[\delta_{\Omega_{n}, 0} G\left(i \omega_{n}, 0\right) G\left(i \omega_{n}^{\prime}, 0\right)\right. \\
& \left.-\delta_{\Omega_{n}, \omega_{n}-\omega_{n}^{\prime}} G\left(i \omega_{n}, x_{L}-x_{R}\right) G\left(i \omega_{n}-i \Omega_{n}, x_{R}-x_{L}\right)\right]
\end{aligned}
$$

where $G(\tau, x)=-\left\langle T_{\tau}\left(\psi(\tau, x) \psi^{\dagger}(0,0)\right)\right\rangle$ is the Green's function for interacting electrons on the ring and $G\left(i \omega_{n}, x\right)$ is its Fourier transform with respect to the imaginary time variable $\tau$. For non-interacting electrons we can apply Wick's theorem and find that this expression is exact. Substituting this into the expression (雨) gives, after proper analytic continuations, the DC conductance

$$
\begin{aligned}
\sigma & \approx \frac{e^{2}}{h} \frac{\left|t_{L} t_{R}\right|^{2}}{\hbar^{2}} D_{L}\left(\epsilon_{F}\right) D_{R}\left(\epsilon_{F}\right) \int_{-\infty}^{\infty} d \omega\left(-\frac{\partial n_{F}(\omega)}{\partial \omega}\right) G^{\mathrm{ret}}\left(\omega, x_{L}-x_{R}\right) G^{\mathrm{adv}}\left(\omega, x_{R}-x_{L}\right) \\
& \approx \frac{e^{2}}{h} \frac{\left|t_{L} t_{R}\right|^{2}}{\hbar^{2}} D_{L}\left(\epsilon_{F}\right) D_{R}\left(\epsilon_{F}\right)\left|G^{\mathrm{ret}}\left(\omega=0, x_{L}-x_{R}\right)\right|^{2}
\end{aligned}
$$


where the last expression is valid if the system is further than $k_{B} T$ from a resonance.

This expression can also be understood using a scattering matrix approach regarding the ring as a (complicated) scatterer for the free electrons in the leads as discussed in a specific case by Jagla and Balseiro [5]. From that point of view our basic approximation is that one scattering event is completed before another one takes place - the approximation breaks down near resonance when the dwell time for extra electrons in the ring is large.

Now we turn to evaluating the retarded Green's function for interacting electrons in a finite ring at a finite temperature. We use the low-energy expansion $\psi(x)=\psi_{+}(x)+$ $\psi_{-}(x)$ where $\psi_{ \pm}(x)$ are clockwise and counterclockwise moving fermions so that $G(\tau, x)=$ $G_{++}(\tau, x)+G_{--}(\tau, x)$. Following Haldane [2], the correlation functions can be evaluated exactly and we obtain $(p= \pm 1)[6]$

$$
\begin{gathered}
\left\langle\psi_{p}(x, \tau) \psi_{p}^{\dagger}(0)\right\rangle=\frac{i}{2 L} e^{-i \frac{p \pi x}{L}} e^{\frac{\pi}{L}\left(N_{0} g^{-1} v+p J_{0} g v\right) \tau} \\
\frac{\vartheta_{3}\left(i g^{-1} \alpha N_{0}-x_{N}, e^{-2 g^{-1} \alpha}\right) \vartheta_{3}\left(i g \alpha p J_{0}-x_{J}, e^{-2 g \alpha}\right)+\vartheta_{2}\left(i g^{-1} \alpha N_{0}-x_{N}, e^{-2 g^{-1} \alpha}\right) \vartheta_{2}\left(i g \alpha p J_{0}-x_{J}, e^{-2 g \alpha}\right)}{\vartheta_{3}\left(i g^{-1} \alpha N_{0}, e^{-2 g^{-1} \alpha}\right) \vartheta_{3}\left(i g \alpha p J_{0}, e^{-2 g \alpha}\right)+\vartheta_{2}\left(i g^{-1} \alpha N_{0}, e^{-2 g^{-1} \alpha}\right) \vartheta_{2}\left(i g \alpha p J_{0}, e^{-2 g \alpha}\right)} \\
\left.\frac{1}{\vartheta_{1}\left(\frac{\pi(i v \tau-p x)}{L}, e^{-\alpha}\right)}\left|\frac{(a / L)}{2 \vartheta_{1}\left(\frac{\pi(i v \tau-p x)}{L}, e^{-\alpha}\right)}\right|\right|^{\gamma}\left|\vartheta_{1}^{\prime}\left(0, e^{-\alpha}\right)\right|^{\gamma+1}
\end{gathered}
$$

where $\gamma=\frac{1}{2}\left(g+g^{-1}\right)-1$ and we introduced the shorthand notation $\alpha=\frac{\pi v \hbar \beta}{L}, x_{N}=$ $\frac{\pi}{L}\left(i g^{-1} v \tau-p x\right)$, and $x_{J}=\frac{\pi}{L}(i g v \tau-p x)$. Here $a$ is a short distance cutoff for the interaction, and is of the order of the lattice spacing. The Jacobi theta functions [7] $\vartheta_{3}$ and $\vartheta_{2}$ arise from the $q=0$ modes with $N$ and $J$ both even or odd, respectively, and the $\vartheta_{1}$ arises from the bosons with $q \neq 0$. The appearance of doubly periodic elliptic functions is natural since the Green's function must be periodic in $x$ and antiperiodic in $\tau$. The Jacobi functions appear also in the partition function of an isolated ring (essentially the $x$ - and $\tau$-independent parts of the above expression) and therefore in most equilibrium properties of mesoscopic rings like persistent currents [8].

The parameters that are most readily accessible in an experiment are the gate voltage and the magnetic flux. They enter only the $q=0$ part of the Hamiltonian which we can re-write as $H_{0}=\frac{1}{2} E_{c}\left(\hat{N}-N_{0}\right)^{2}+\frac{\hbar v_{F}}{2 L}\left(\hat{J}-J_{0}\right)^{2}$ where $v_{F}=g v$ is the Fermi velocity of a non-interacting system with the same density and $E_{c}=\frac{\pi v_{F}}{g^{2} L}$ is the charging energy. The conductance resonances correspond to values of the gate voltage and magnetic flux at which the ground state quantum numbers $N$ and $J$ change (degenerate ground state). Hence, the positions of conductance resonances can be determined from a simple charging energy model with a single-particle Aharonov-Bohm term - note, however, that $E_{c}$ is not simply given by the geometric capacitance (it is non-zero even for non-interacting electrons). In the $\left(V_{g}, \Phi\right)$-plane the resonance positions form a network the shape of which depends on the interaction parameter $g$. We suggest therefore that the interaction parameter can be experimentally measured by studying the trajectories of conductance maxima as a function of the gate voltage and magnetic flux. For non-interacting systems the resonance positions form a lattice of diamond shaped parallelograms, whereas for repulsive interactions $(g<1)$ there are some values of the gate voltage such that a resonance condition is not met for any $\Phi$ as indicated in Figure 1. For attractive interactions, there are ranges of $\Phi$ such that the total current in the ring, $J$, is independent of $V_{g}$. In that case electrons can tunnel into and out of the ring only as pairs of clockwise- and counterclockwise movers, which is reminiscent of Cooper pair tunneling through a superconducting grain [9]. From now on we 
only consider repulsive interactions.

We use the expressions (5) and (6) to analyze the dependence of the conductance on the external parameters. The dependences on $V_{g}$ and $\Phi$ are qualitatively similar and in Figure 22 we show the conductance as a function of $V_{g}$. Near a resonance we obtain the limiting behavior $\left|G^{\mathrm{ret}}(0, x)\right|^{2} \sim C /(\delta \epsilon)^{2}$ where $\delta \epsilon$ is the energy cost of changing the number of electrons in the ring by one (we still assume $\delta \epsilon>k_{B} T$ ). In a generic case a resonance corresponds to a degeneracy for the addition or removal of either a clockwise or counterclockwise moving particle, and the prefactor $C$ is independent of device geometry. However, since we have two control parameters $V_{g}$ and $\Phi$, we can use them to bring both clockwise and counterclockwise modes to resonance simultaneously. These special parameter values correspond to slope changes in the trajectories of conductance resonances in Figure 1. Since at these double resonances the Green's function has significant contributions from wave vectors $k_{F+}$ and $-k_{F-}$, its absolute square has components with wave vector $k_{F+}-\left(-k_{F-}\right)=2 \pi N / L$. Thus, we find that near a double resonance the amplitude of the conductance maximum varies periodically with the separation between the tunnel junctions as $\left(1+\cos \left[\frac{2 N \pi}{L}\left(x_{L}-x_{R}\right)\right]\right)$. Away from a double resonance one channel dominates and the amplitude of these oscillations is exponentially small. Since the wave vector of these oscillations is essentially $2 k_{F}$, they can be observed only in low-density systems or using local probes like STM. The interference effects are smeared out by temperature which leads to a different temperature dependence of the conductance for different device geometries near a double resonance: if $N\left|x_{L}-x_{R}\right| \approx n L$, where $n$ is an integer, the conductance decreases with temperature due to reduced interference, whereas for $N\left|x_{L}-x_{R}\right| \approx\left(n+\frac{1}{2}\right) L$ the conductance increases with $T$.

From the expression (6) we see that the conductance is reduced due to interactions by a factor $(a / L)^{2 \gamma}$ which can be attributed to an orthogonality catastrophe that has previously been studied in the context of quantum dots in the fractional quantum Hall regime [10. The exponent governing the resonance line shape for small $\delta \epsilon, \sigma \sim 1 /(\delta \epsilon)^{2}$, is independent of the interaction parameter $g$, which is a consequence of a finite minimum energy of the bosonic modes. The resonant contribution dominates for $\delta \epsilon \lesssim \delta \epsilon_{c}=\frac{2 \pi \hbar v}{L}\left|\sin \left(\frac{\pi\left(x_{L}-x_{R}\right)}{L}\right)\right|^{\gamma}$ (up to logarithmic corrections); for $\delta \epsilon \gg \delta \epsilon_{c}$ the valley conductance levels off to a constant value proportional to $\left(a /\left|x_{L}-x_{R}\right|\right)^{2 \gamma}$ as seen in Figure 6. For large separations $\Delta x=\left|x_{L}-x_{R}\right|$ the crossover point $\delta \epsilon_{c}$ exceeds half of the resonance spacing and the crossover is not observed. The two limiting behaviors can be combined to give the approximate line shape

$$
\sigma(\delta \epsilon) \sim \Gamma_{L} \Gamma_{R}\left[\frac{1}{(\delta \epsilon)^{2}}\left(\sqrt{2} \frac{a}{L}\right)^{2 \gamma}+\left(\frac{L}{2 \pi \hbar v}\right)^{2}\left|\frac{L}{\sqrt{2} a} \sin \left(\frac{\pi \Delta x}{L}\right)\right|^{-2 \gamma}\left(\frac{1-e^{\gamma \log \left|\sin \left(\frac{\pi \Delta x}{L}\right)\right|}}{\gamma}\right)^{2}\right]
$$

where $\gamma=\frac{1}{2}\left(g+g^{-1}\right)-1$ and $\Gamma_{L / R}=\left|t_{L / R}\right|^{2} D_{L / R}\left(\epsilon_{F}\right)$ are the line widths for a non-interacting system. The last factor gives rise to a logarithmic dependence on $\Delta x$ in the non-interacting limit.

At $T=0$ the smallest $\delta \epsilon$ that we can consider is determined by when terms that are higher order in the tunneling Hamiltonian become significant. Due to the orthogonality catastrophe that happens when the first term in (7) is of order unity. Therefore, we expect 
that at $T=0$ the height of the resonance peak is independent of $g$, and the peak width is given by $\delta \epsilon$ such that $\Gamma_{L} \Gamma_{R}\left(\sqrt{2} \frac{a}{L}\right)^{2 \gamma}(\delta \epsilon)^{-2} \approx 1$. A simple Breit-Wigner formula $\sigma \sim \frac{\Gamma^{2}}{(\delta \epsilon)^{2}+\Gamma^{2}}$ gives a peak-to-valley ratio $2(\Gamma / \Delta)^{2}$ where $\Delta$ is the separation between adjacent resonances and $\Gamma$ is the resonance width. In the present case this simple connection does not hold: the line width at $T=0$ is reduced by a factor $(a / L)^{\gamma}$ whereas the valley current (for small $\Delta x$ ) is only suppressed by factor $(a / \Delta x)^{2 \gamma}$. The former suppression factor can be identified as the life time of a charged excitation of the ring while the second one is the off-resonance probability of transmission through the ring. The valley current is therefore anomalously large for small $\Delta x$ since effectively the system size is replaced by $\Delta x$ and the orthogonality catastrophe is less severe. Since at $T=0$ the peak widths are reduced by interactions, the resonance peak heights at a finite temperature are suppressed due to thermal broadening. Thus, the main effect of interactions is to change the peak-to-valley ratio in a way that depends on device geometry and temperature.

The experimental possibilities for the study of nanostructures like the one we consider are developing rapidly. New techniques like conducting organic molecules and carbon compounds are emerging to complement the conventional semiconductor structures. In particular, it was recently demonstrated [11] that carbon nanotubes exhibit coherent electron transport and can be used to fabricate nanoscale ring structures. We believe these devices can be used to experimentally study the system we have analyzed.

In conclusion, we have considered tunneling through a finite strongly interacting system within the framework of an exactly solvable model. We find that the positions of conductance resonances in the $\left(V_{g}, \Phi\right)$-plane can be used to determine the interaction parameter $g$. We conclude that at $T=0$ the heights of resonance peaks are unaffected by interactions but due to the narrowness of $T=0$ resonances, the peak conductance at a finite temperature is reduced by interactions. The valley current depends on both interactions and the device geometry. Near a double resonance we find that the heights of resonance peaks depend on device geometry due to interference between different current carrying processes. 


\section{REFERENCES}

[1] G. D. Mahan, Many Particle Physics, (Plenum, New York, 1990).

[2] F. D. M. Haldane, J. Phys. C 14, 2585 (1981).

[3] C. L. Kane and M. P. A. Fisher, Phys. Rev. Lett. 68, 1220 (1992).

[4] R. Fazio, F. W. J. Hekking, and A. A. Odintsov, Phys. Rev. Lett. 74, 1843 (1995).

[5] E. A. Jagla and C. A. Balseiro, Phys. Rev. Lett. 70, 639 (1993).

[6] For details see A. Mattsson, S. Eggert, and J. M. Kinaret, unpublished (1997).

[7] I. S. Gradshteyn and I. M. Ryzhik, Table of Integrals, Series, And Products, (Academic, Orlando, 1980).

[8] D. Loss, Phys. Rev. Lett. 69, 343 (1992).

[9] K. A. Matveev et al., Phys. Rev. Lett. 70, 2940 (1993).

[10] J. M. Kinaret et al., Phys. Rev. B 45, 9484 (1992); ibid, 46, 4681 (1992).

[11] J. Liu et al., Nature 385, 780 (1997); S. J. Tans et al., ibid. 386, 474 (1997). 


\section{FIGURES}

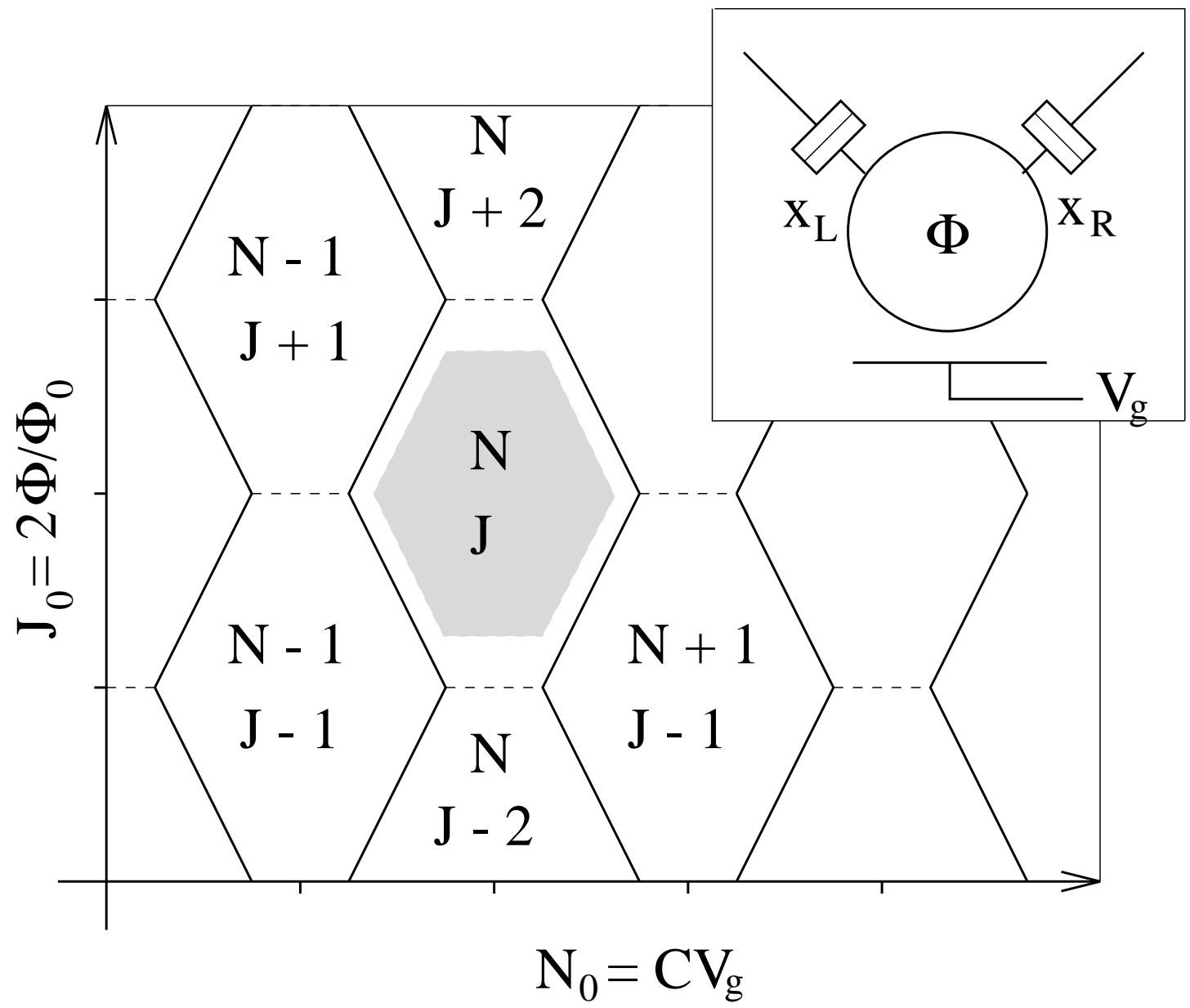

FIG. 1. Positions of conductance resonances in the $\left(V_{g}, \Phi\right)$-plane for interacting electrons (repulsive interactions, $g=1 / \sqrt{2}$ ). The labels $N$ and $J$ denote the ground state charge and current as a function of the external parameters, and the shaded area indicates the domain of validity of our analysis. The line segments with different slopes correspond to fluctuations in the numbers of clockwise and counterclockwise moving electrons, respectively. Inset: device geometry. 


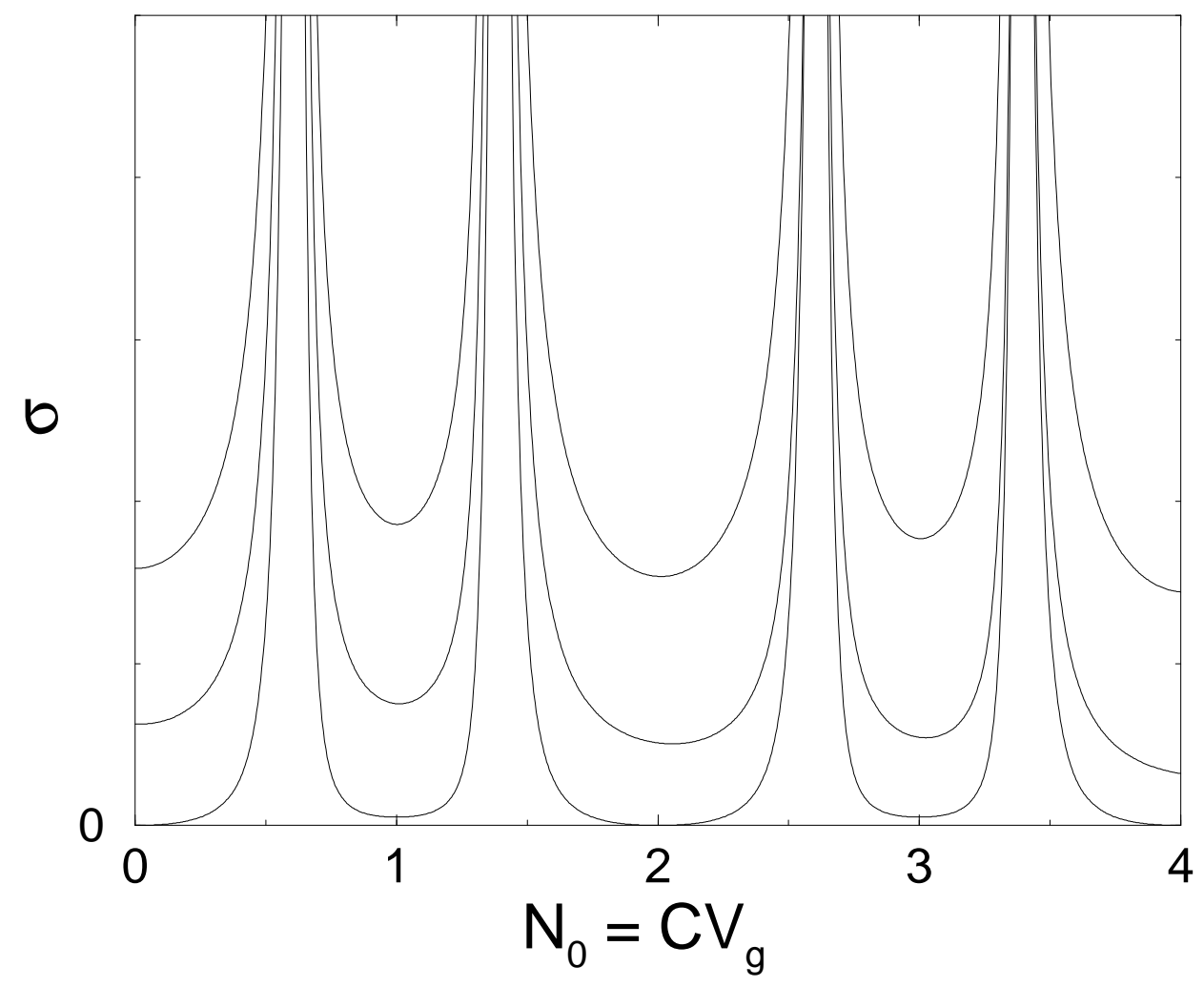

FIG. 2. Conductance vs. $V_{g}$ for $x_{L}-x_{R}=0.02 L, x_{L}-x_{R}=0.05 L$ and $x_{L}-x_{R}=0.5 L$ (from top to bottom). The temperature is $T=0.1 \frac{\hbar v}{L}$ and the interaction parameter is $g=1 / 2$. 


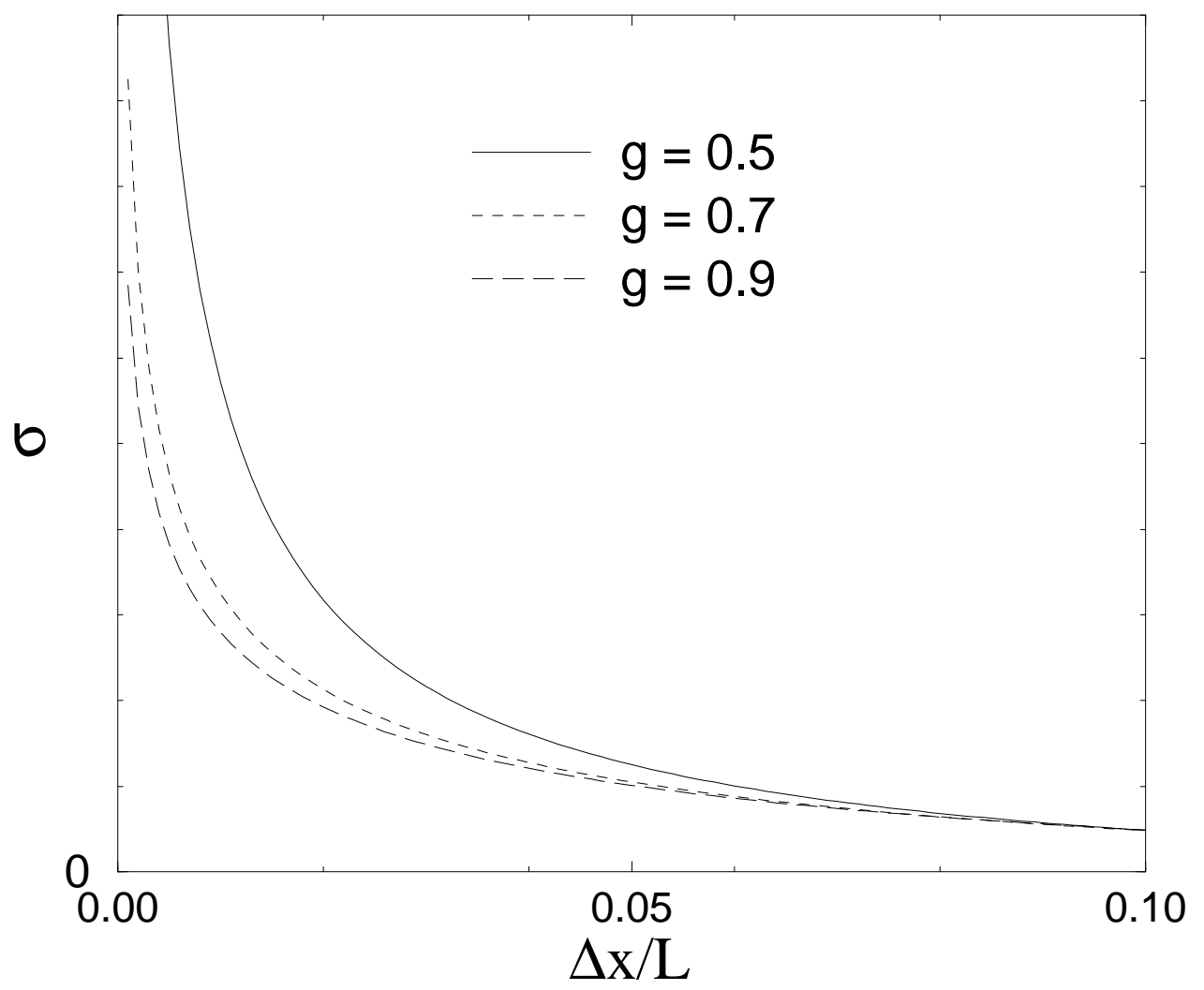

FIG. 3. Conductance vs. $\left|x_{L}-x_{R}\right|$ near a conductance minimum for interaction parameters $g=0.9, g=0.7$, and $g=0.5$. The temperature is $T=0.1 \frac{\hbar v}{L}$. 